\title{
Remembering to Learn: Independent Place and Journey Coding Mechanisms Contribute to Memory Transfer
}

\author{
Amir S. Bahar and Matthew L. Shapiro \\ Fishberg Department of Neuroscience and Alfred B. and Gudrun J. Kastor Neurobiology of Aging Laboratories, Mount Sinai School of Medicine, New York, \\ New York 10029-6574
}

The neural mechanisms that integrate new episodes with established memories are unknown. When rats explore an environment, CA1 cells fire in place fields that indicate locations. In goal-directed spatial memory tasks, some place fields differentiate behavioral histories (“journey-dependent” place fields) while others do not (“journey-independent" place fields). To investigate how these signals inform learning and memory for new and familiar episodes, we recorded CA1 and CA3 activity in rats trained to perform a "standard" spatial memory task in a plus maze and in two new task variants. A "switch" task exchanged the start and goal locations in the same environment; an "altered environment" task contained unfamiliar local and distal cues. In the switch task, performance was mildly impaired, new firing maps were stable, but the proportion and stability of journey-dependent place fields declined. In the altered environment, overall performance was strongly impaired, new firing maps were unstable, and stable proportions of journey-dependent place fields were maintained. In both tasks, memory errors were accompanied by a decline in journey codes. The different dynamics of place and journey coding suggest that they reflect separate mechanisms and contribute to distinct memory computations. Stable place fields may represent familiar relationships among environmental features that are required for consistent memory performance. Journey-dependent activity may correspond with goal-directed behavioral sequences that reflect expectancies that generalize across environments. The complementary signals could help link current events with established memories, so that familiarity with either a behavioral strategy or an environment can inform goal-directed learning.

\section{Introduction}

Episodes occur in the context of established memories that influence subsequent recall, but the neuronal mechanisms that integrate learning and knowledge are unknown. Learning new facts, recalling recent events, and imagining the future require the hippocampus (Tulving and Markowitsch, 1998; Ekstrom et al., 2003; Squire et al., 2004; Schacter and Addis, 2009). Human hippocampal neurons respond selectively to specific words and faces, during specific experiences, immediately before events are recalled (Gelbard-Sagiv et al., 2008), and in anticipation of familiar sequences (Paz et al., 2010). Hippocampal damage in rats impairs learning about places and remembering recent events, and rat hippocampal neurons fire in place fields defined by location and modulated by tasks (O'Keefe and Nadel, 1978; Markus et al., 1995; Deadwyler et al., 1996; Moita et al., 2003; Lenck-Santini et al., 2008). Preventing the establishment of stable place fields impairs spatial learning (Shapiro and O’Connor, 1992; Kentros et

\footnotetext{
Received July 29, 2011; revised Dec. 12, 2011; accepted Dec. 15, 2011.

Author contributions: A.S.B. and M.L.S. designed research;A.S.B. performed research; A.S.B. and M.L.S. analyzed data; A.S.B. and M.L.S. wrote the paper.

This work was supported by the Mount Sinai School of Medicine and the National Institutes of Health Grants MH065658 and MH073689. A.S.B. was supported in part by a 2008 National Alliance for Research on Schizophrenia and Depression Young Investigator award. We thank Maojuan Zhang for technical assistance. We thank Howard Eichenbaum, Kevin Guise, Mark Baxter, Sam McKenzie, Yuji Naya, Justin Riceberg, and Wendy Suzuki for their comments on earlier drafts of the manuscript.

Correspondence should be addressed to Dr. Matthew L. Shapiro, Mount Sinai School of Medicine, One Gustave Levy Place, Box 1065, New York, NY 10029. E-mail: matthew.shapiro@mssm.edu.

DOI:10.1523/JNEUROSCI.3998-11.2012

Copyright $\odot 2012$ the authors $\quad 0270-6474 / 12 / 322191-13 \$ 15.00 / 0$
}

al., 1998), and disrupting firing patterns impairs memory (e.g., Hampson and Deadwyler, 2000; Robbe and Buzsáki, 2009).

In goal-directed memory tasks, place fields distinguish overlapping spatial journeys between different start and goal locations (Frank et al., 2000; Wood et al., 2000; Ferbinteanu and Shapiro, 2003; Bower et al., 2005; Smith and Mizumori, 2006; Ainge et al., 2007). Journey-dependent place field activity ("journey coding”) correlates with recent memory demand (Smith and Mizumori, 2006) and declines during memory errors (Ferbinteanu and Shapiro, 2003), but its contribution to learning and memory remains unclear. Journey coding could represent temporally extended episodes that guide specific behaviors (Ferbinteanu et al., 2006) but may not generalize across situations or guide new learning. Or, journey coding could help organize subsequent learning by representing general features in memory (e.g., either the "facts" that a maze has distinct start and goal arms connected by a choice point), the rules and procedures that guide behavior (e.g., that the most recently rewarded goal is most likely to contain food now), or generic episodic structure (e.g., signals that frame the beginning and end of behavioral episodes, analogous to start and stop sequences in gene transcription). Alternatively, it could be a consequence of regular trajectories and reflect, rather than guide, behavior.

To test these possibilities, we recorded CA1 and CA3 ensembles as rats learned and performed hippocampus-dependent memory tasks in a plus maze (Fig. 1; Bahar et al., 2011). After training to perform a standard (STD) matching-to-place task with serial reversals, the rats were presented with two task vari- 
ants that followed the same rules but required different discriminations. In a switch (SW) task, the start and goal locations were interchanged, so the rat had to adopt opposite spatial trajectories in the same environment. In an altered environment (ALT) task, the STD procedures were used, but local and distal cues were unfamiliar. The same neuronal populations were recorded in two sessions each day: in one the rats performed the STD task, in the other they performed one of the task variants. By comparing unit activity during identical spatial behaviors in the different tasks, the present experiment investigated how journey coding correlated with memory-guided learning.

\section{Materials and Methods}

The subjects, data collection methods, and behavioral analyses were identical to a previous report on place field dynamics (Bahar et al., 2011) but not journey coding, which is described here.

Subjects. Male Long-Evans rats (Charles River Laboratories) weighed $\sim 350$ g, were maintained at $85 \%$ of their free-feeding weight, and were housed individually on a $12 \mathrm{~h}$ light/ dark cycle. Training and testing were restricted to the dark phase. All experiments and surgical procedures were in accordance with the guidelines of the Mount Sinai Institutional Animal Care and Use Committee and the NIH.

Apparatus. A recording room $(2.8 \mathrm{~m} \times$ $3.2 \mathrm{~m}$ ) contained a gray, wooden plus maze elevated $82 \mathrm{~cm}$ above the floor with arms $66 \mathrm{~cm}$ long, $6.1 \mathrm{~cm}$ wide, and walls $2.5 \mathrm{~cm}$ high. The distal end of each arm had a recessed food well ( $1 \mathrm{~cm}$ diameter) that contained a Froot Loop made unavailable by a wire screen. A wooden block prevented entrance to the unused start arm. A rectangular waiting platform $(30 \mathrm{~cm}$ wide, elevated $100 \mathrm{~cm}$ ) was located in the southeast corner (Fig. $1 A$ ). The room contained several visual cues e.g., posters. In the altered environment task, black curtains and new visual cues covered the walls, the maze surface was covered with brown plastic, and a new wooden block and waiting platform were used. The new waiting platform was placed at the northeast corner. The location and orientation of the maze in the room was not changed.

Recording. Twelve independently movable tetrodes made of four twisted $12.7 \mu \mathrm{m}$ in diameter nickel-chromium wires (RO-800; Kanthal Precision Technology) were mounted in a Neuro-hyper-drive assembly (Kopf Instruments). Assemblies were connected to a headstage with source follower amplifiers (Kopf 54, Neuralynx). Ten color LEDs on the headstage were detected by an overhead color LCD camera to track position and heading $(640 \times 480$ pixel resolution at $60 \mathrm{~Hz})$. Neural signals were amplified 2000-5000 times, filtered between 0.6 and $6 \mathrm{kHz}$, digitized at $32 \mathrm{kHz}$, and stored together with tracking information on a computer (DigitalLynx4S, Neuralynx).

Behavioral training and testing. Standard task, STD, is shown in Figure $1 \mathrm{~A}$. Rats were handled ( $15-30 \mathrm{~min} / \mathrm{d}$ ) for $3 \mathrm{~d}$ and then allowed to explore the maze for $2 \mathrm{~d}$. For the STD task, the North and the South arms were used as start arms; the West and the East were goal arms. Before each trial, one-fourth of a Froot Loop was placed in the well at the end of one goal arm. In each trial the rat was placed on the end of one of the start arms facing the center and had to enter the correct goal arm to find the food. Entering the unrewarded arm for $\geq 1$ body length was recorded as an error; the rats were allowed to correct their choices. The start arm was selected pseudorandomly so that the same arm was not repeated $>3$ consecutive times. Between trials the rat was placed on the waiting platform for 15-20 s. After achieving 9/10 correct trials, the goal was reversed and a new block of trials began. Each session was composed of four blocks. The switch task, SW, was introduced for the first time during recording sessions using procedures that were identical to the STD, but the East and West arms were used as start arms and the North and South arms were used as goal arms. The altered environment, ALT, was also introduced for the first time during recording sessions. New local and distal cues altered the room as described above. All other procedures were identical to the STD task. Four rats performed the SW task, and 6-7 d later the ALT task. Two rats performed the ALT task first and the SW task later. The task order did not affect firing patterns.

Surgery and tetrode placement. Rats were anesthetized with isoflurane and placed in a stereotaxic frame. The tetrode assembly was fixed to the skull with dental cement and the tetrodes were implanted above the left dorsal hippocampus (anteroposterior, $3.8 \mathrm{~mm}$; mediolateral, $3 \mathrm{~mm}$ from bregma). Two ground wires were attached to skull screws. After the surgery, tetrodes and two reference wires were lowered $1 \mathrm{~mm}$ into the cortex and rats were injected with Banamine (flunixin meglumine, 1.5 $\mathrm{mg} / \mathrm{kg}$, i.m.). When simultaneously recording from CA1 and CA3, six of the tetrodes located laterally were lowered to CA3, the rest were lowered to CA1. Reference wires were lowered to the corpus callosum. Rats were allowed to recover for $6-8 \mathrm{~d}$ before behavior testing resumed.

Unit isolation and screening. Single units with $\geq 3: 1$ signal-to-noise ratios and peaks $>100 \mu \mathrm{V}$ were discriminated offline. Complex spike waveforms were characterized by $8-32$ parameters (e.g., peak amplitudes, etc.). A custom computer program displayed waveform parameter 
values as points in a multidimensional space. Points were assigned to distinct clusters using a semiautomated, nonlinear, cluster-cutting algorithm (Ferbinteanu and Shapiro, 2003). The discrimination was further quantified by Mahalanobis distance between clusters in a normalized parameter space. Any pair of clusters $<2$ standard deviations apart were flagged, and only units with minimal overlap with neighboring clusters and noise were included in subsequent analyses. Putative interneurons (i.e., fast spiking, short-duration waveforms) were not analyzed.

Waveform stability. To maximize recording stability, tetrodes were advanced at a rate of $20-30 \mu \mathrm{m} / \mathrm{d}$ after reaching $\sim 200 \mu \mathrm{m}$ above the layer (based on the amplitude of sharp waves and ripples in the slowwave signal). Recording sessions began after tetrodes settled for at least $72 \mathrm{~h}$. Electrode stability was assessed offline by comparing waveforms and cluster distributions qualitatively and quantitatively. Units identified in one session were compared with all other sessions visually and matched based on waveform similarity, cluster location, size, and boundaries (see Fig. 2D). Mahalanobis distances were computed between the clusters. Distance $>2$ standard deviations confirmed cluster separation whereas distance $<1$ standard deviation suggested overlap, although all cases were visually inspected to ensure separation and consistency across different sessions. Peak and trough amplitudes ( $1 \mathrm{~ms}, 32$ measures) were extracted from the averaged waveforms and compared by Pearson's $r$ (Kennedy and Shapiro, 2009). The $r$ values were $\geq 0.90$ between the first and last recording sessions (Fig. $1 B$ ). Data acquired from tetrodes that showed altered waveforms between recording sessions were excluded from further analysis.

To further test neuronal stability, we compared waveforms and place fields recorded from the same cell populations in a separate random foraging task (Muller and Kubie, 1987) before and after each experiment. An open square box made of opaque Plexiglas ( $80 \mathrm{~cm} / \mathrm{side}, 39 \mathrm{~cm}$ high) with different visual cues on each wall was elevated $85 \mathrm{~cm}$ from the floor and placed in the center of the testing room. Unit recording began after the rats were familiar with the box and searched readily for chocolate sprinkles. We recorded unit activity in the random foraging task in two 10 min sessions, once before the first STD recording session on day 1 and again a few minutes after the last SW or ALT sessions on day 2. More than $76 \%$ of CA1 and CA 3 cells had stable place fields in the box in both random foraging sessions. The mean spatial correlation and the median population vector correlation were $\geq 0.6$ between the first and last session in both CA1 and CA3, consistent with previous quantitative studies (Kentros et al., 1998; Leutgeb et al., 2004). The results provide converging evidence that the same populations of cells were recorded throughout the two recording days.

Maze behavior. Video tracking data were used to quantify behavior on the maze. The instantaneous location of the rat's head, signaled by LEDs on the headstage, was time stamped and stored in computer files for each session. Post hoc analyses assigned camera pixels to grid units $(6 \times 6 \mathrm{~cm})$. Recordings were separated into four journeys (North-to-West, North-toEast, South-to-West, and South-to-East during STD and ALT sessions, and West-to-North, West-to-South, East-to-North, and East-to-South during SW sessions). Only data recorded in areas restricted to maze arms were analyzed and included $70-90 \%$ of each arm where the movement of the rat was reliable. The number of visits, dwell time, heading direction, and running speed were calculated for each grid unit, and the reliability of behavior across different trials was quantified using paired $t$ tests and Pearson's r. A significant difference $(p<0.05)$ of any of these parameters excluded the data from further analyses. All analyses excluded activity in the central area of the maze and the waiting platform, where behavior was likely different across trials.

Place fields. Firing rate was calculated for each grid unit by dividing the number of spikes by the time spent in each (filtered at speed $>2 \mathrm{~cm} / \mathrm{s}$ ). Cells with a mean firing rate $\geq 1$ spike/s and grid units occupied for $\geq 300$ $\mathrm{ms}$ and visited $\geq 4$ times in a session were analyzed for place field activity. A place field was defined as an area of $\geq 2$ adjacent grid units with mean firing rate $\geq 1$ spike/s, $\geq 3$ spikes/subfield visit, and a firing probability of $>30 \%$ across trials. The analyses included only the restricted areas and only correct trials unless noted otherwise. Place fields were analyzed separately for each journey.
Journey-dependent activity. If a place field was defined in a journey, then journey-dependent activity was quantified by comparing overlapping paths through the same arm, i.e., in a start arm on the way to different goal or in a goal arm on the way from different starts (e.g., in the $\mathrm{N}$ arm during NE vs NW journeys) (see Fig. 3). We refer to these overlapping paths as "corresponding journeys." Pearson's $r$ and Student's $t$ tests compared the distribution of firing rates in the arrays of grid units from these corresponding journeys. The $t$ test measured the magnitude of the mean firing rate differences between corresponding journeys: a high $t$ shows different firing rates and hence journey-dependent activity. A high $r$ shows similar distributions of firing rates across the grid units in corresponding journeys, i.e., journey independence, whereas a low correlation indicates more distinct firing patterns, i.e., journey dependence. If the rate maps differed by a paired $t$ test or did not differ by Pearson's $r$, then the place field was defined as "journey dependent." If a cell was entirely silent in one journey type (i.e., a null matrix of grid units), Pearson's $r$ is undefined and was assigned a value of 0 . The proportions of categorically defined journey-dependent and journey-independent place fields were compared across different sessions using $\chi^{2}$ statistics.

Journey-dependent coding in a start arm defined prospective coding; in a goal arm it defined retrospective coding (Ferbinteanu and Shapiro, 2003). Journey coding was tested independently for each task and recording session. Additional correlation analysis compared the firing rate of each cell across corresponding journeys using the mean firing rate in each arm as a whole, collapsing across grid units (see Fig. $4 C-F$ ). As described in the paragraph above, a significant increase in correlation between journeys indicates reduced journey coding. To determine whether journey coding was stable across tasks, we assigned each cell to a coding category and counted the number of transitions from the first STD to either the first SW or ALT session. Three coding categories and two tasks define nine transitions $\left[\left(n^{2}-n\right) / 2\right]$, i.e., prospective to prospective (PP), prospective to retrospective (PR), independent to retrospective (IR), etc. If the coding category was a fixed property of cells, then, e.g., prospective activity in the STD should predict prospective activity in the SW. If journey coding was determined functionally, e.g., by plastic network properties, then the categories should be independent among tasks. To estimate the distribution expected by random assignment, we multiplied the proportion of place fields in a given category observed in the new task by the proportion of the comparison category observed in the standard. For example, Figure $4 \mathrm{~A}$ shows that 0.27 of all place fields are categorized as prospective in STD1 compared to 0.12 in SW1. The proportion of place fields expected to be categorized as prospective in both tasks would be the product of the two, 0.03 .

Activity dynamics. To assess coding stability, spatial and journeyrelated activities were compared within and between sessions (e.g., in the first and second half of a SW session and in repeated STD and repeated SW sessions). Correlation analyses compared the firing rates of the same recorded neurons in each maze arm across corresponding pairs of journeys (Fig. 4). Changes to journey related activity within and between conditions was measured by comparing these correlations using standard methods. Each Pearson's $r$ was transformed to Fisher's $Z$, the $Z$ of one condition subtracted from the other and the difference divided by the degrees of freedom $\left(Z=\left(r 1^{\prime}-r 2^{\prime}\right) / \operatorname{sqrt}([1 /(N 1-3)]+1 /[N 2-\right.$ $3]$ ), where sqrt is the square root) (SYSTAT Software, 2007). Error trials were analyzed by comparing the firing rate maps constructed from blocks of 4-5 trials on a journey-by-journey basis. For example, journeydependent activity in the North start identified during correct trials would be compared during incorrect trajectories to the Northeast or Northwest. Error trials were compared to correct trials only if the movement of the rat was statistically indistinguishable between the conditions. Place field stability across repeated sessions independent of journeys was quantified by comparing firing maps averaged across the entire recording session (Bahar et al., 2011).

Histology. Rats were anesthetized with sodium pentobarbital (100 mg/ $\mathrm{kg}$, i.p.). A DC current was passed through each tetrode ( $40 \mu \mathrm{A}, 4 \mathrm{~s})$ to induce a lesion. Brain sections ( $35 \mu \mathrm{m}$ ) were labeled with formol-thionin stain (see Fig. 2C). 


\section{Results}

Learning was facilitated by prior training

The behavioral results were described in detail previously (Bahar et al., 2011). The rats learned the STD task in 6-7 daily sessions, maintained high performance after surgery, and learned the ALT and SW tasks within 1-2 sessions (Fig. 2A). Criterion performance was maintained in the first SW session, but accuracy declined compared to the STD (mean percentage correct: STD $1=88 \%$, SW $1=82 \%$, $F_{(3,18)}=3.8, p=0.02$, Tukey's post test; Fig. $2 B$ ). Memory accuracy fell below criterion in the first ALT session (mean percentage correct: ALT1 $=69 \%$; STD1 vs ALT1: $\left.F_{(3,12)}=4, p=0.03\right)$, and returned to STD levels in the second session. Thus, the rats acquired the new tasks more rapidly after training in the STD and adopted new spatial trajectories in a highly familiar environment more rapidly than they learned new spatial discriminations.

\section{Place coding remapped between tasks}

"Place coding" refers to the spatial information provided by place fields independent of other behavioral variables. We previously reported spatial coding dy- namics within and between the tasks that analyzed place field maps but not journey-related activity (Bahar et al. 2011). CA1 and CA3 unit activity (Fig. $2 C)$ was recorded as rats $(n=7)$ performed the STD task and either the SW or ALT. Identified waveforms were stable throughout the two days of recording (Fig. $2 D)$, as determined by visual inspection of spike clusters, calculations of Mahalanobis distances between spike clusters, comparisons of spike waveforms with Pearson's $r$, and place field stability across random foraging sessions before and after each experiment (Bahar et al., 2011). Units with stable waveforms included 149 CA1 (four rats) and 100 CA3 cells (four rats) recorded during the STD-SW experiments, and 227 CA1 (four rats) and 72 CA3 cells (three rats) recorded during the STD-ALT experiments. CA1 and CA3 units were recorded simultaneously in two rats. Place fields in both CA1 and CA3 were consistent across repeated STD sessions. In the SW, both neural populations immediately established new place fields that were stable across repeated SW sessions. In the ALT task, new CA1 place fields appeared immediately but were unstable between repeated ALT sessions, and most CA3 place fields vanished (Bahar et al., 2011). Both CA1 and CA3 place fields showed extensive and simultaneous population and rate remapping in the new tasks (Bahar et al. 2011).

\section{Journey coding was prominent in both CA1 and CA3}

The present experiment investigated journey coding, information about behavioral history provided by journey-dependent place field activity, in the same behavioral conditions as described above. We compared unit activity as rats took identical spatial paths in either the same start arm on the way to different goals or in the same goal arm approached from different starts (Fig. 1C). Spatial locations, movement speed, and heading direction were statistically indistinguishable across these corresponding journeys, so that differences in unit activity could be attributed to
B

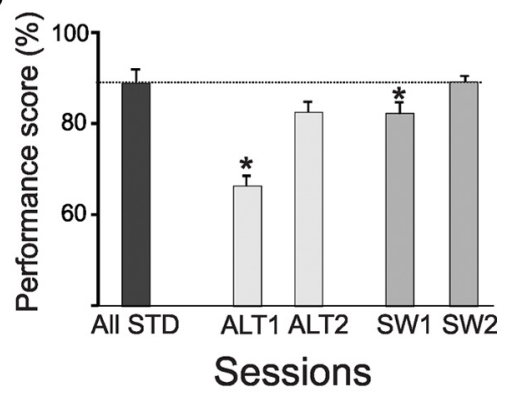

D

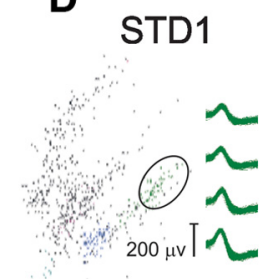

SW2

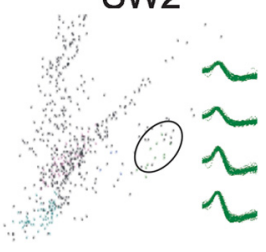

Figure 2. Memory performance and hippocampal recording. $A$, The number of trials to criterion (80\%) was significantly greater and STD2) than during different tasks (e.g., STD1 and SW2). The clusters surrounded by the ellipses show spikes recorded from the SW2 than STD1 (see representative waveforms).

different behavioral histories. Journey-dependent CA1 place fields were common and stable across repeated STD sessions, and retrospective activity was more common than prospective, as observed previously (Fig. 3B; Frank et al., 2000; Ferbinteanu and Shapiro, 2003; Smith and Mizumori, 2006). A new observation was that CA3 neurons also fired in journey-dependent place fields and had the same distribution of prospective and retrospective place fields as CA1 (Fig. 3C).

\section{Journey coding declined in the SW}

We predicted that journey coding would be required for recent memory performance, and that its prominence should correlate with overall memory performance (Ferbinteanu et al., 2006). If journey coding predicted memory performance linearly, then the slight decline in SW performance should correlate with a slight decline in journey coding. The proportion of journey-dependent CA1 place fields decreased markedly, however, from $54 \%$ of all place fields recorded in the STD to $28 \%$ of all place fields recorded in the SW $\left(\dot{\chi}_{(2)}=9.7, p=0.008\right)$ (Fig. $\left.4 A\right)$. Across individual animals, the number of journey-dependent CA1 place fields decreased $34-59 \%$ in the SW compared to the STD. The proportion of journey-dependent place fields also shrank in CA3, but not as reliably (from $58 \%$ in the STD to $34 \%$ in the SW (STD1 vs SW 1 : $\hat{\chi}_{(2)}=5.7, p=0.06$; Fig. $4 B$ ). The overall population of place fields also distinguished journeys more strongly during the STD than SW sessions. In both CA1 and CA3, the spatial correlations between corresponding journeys was lower during the STD than the SW (Pearson's $r$, mean \pm SEM: CA1, STD $1=0.35 \pm 0.05$, $\mathrm{SW} 1=0.66 \pm 0.03, F_{(3,314)}=6.2, p<0.001$; CA3, STD $1=$ $0.43 \pm 0.06, \mathrm{SW} 1=0.6 \pm 0.05, F_{(3,186)}=2.6, p=0.05$; Fig. $4 C-F)$. The same general pattern of results was obtained when the mean firing rates were calculated for each maze arm as a whole, collapsing across grid units (CA1: STD1, $r=0.6$, SW1, $r=$ 
A
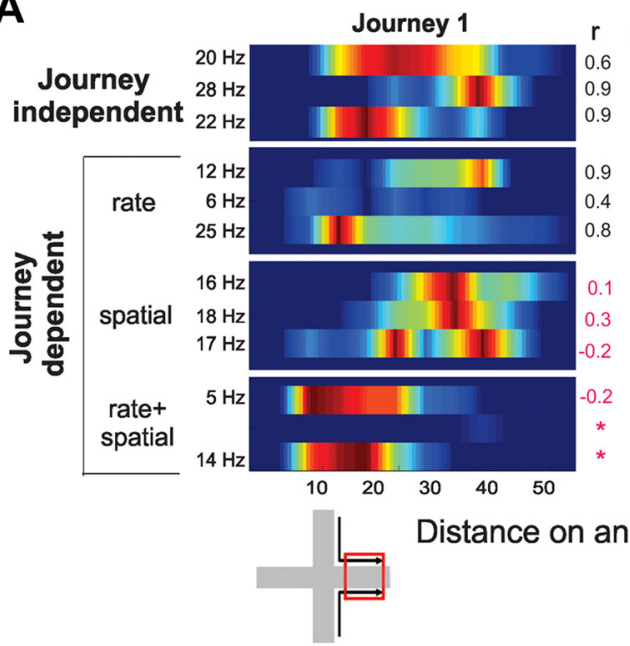

Distance on an arm (cm)

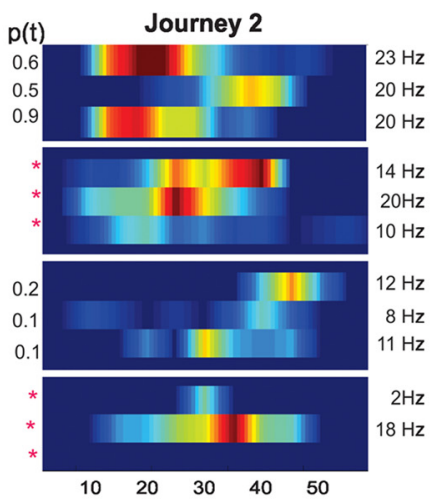

B

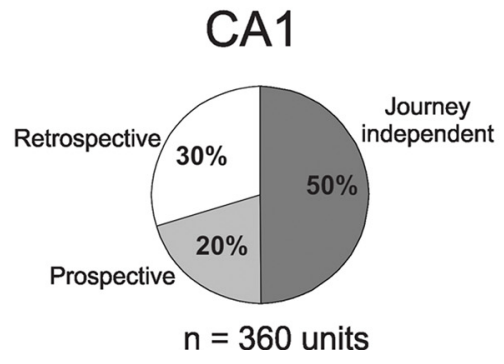

C

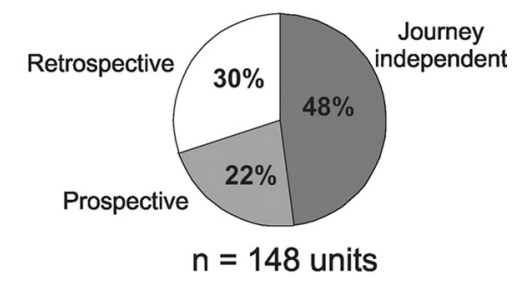

Figure 3. Firing rate maps and journey-dependent activity during STD sessions. $\boldsymbol{A}$, Color-coded firing rate maps compare examples of single unit activity across corresponding journeys ( 1 and 2, e.g., in the East arm, left inset). Each horizontal bar shows the mean firing rate of one cell along one arm during a STD session; adjacent bars show the same cell on the same arm during corresponding journeys. Rate maps, smoothed here but not in statistical analyses, show firing rates normalized to each cell's maximum in each journey (listed at the edge of each panel). Pearson's $r$ (center left) and Student's $t$ ( $p$ value, center right) compared the distributions across journeys, with significant differences shown in red. The top panel shows three cells with journey-independent place fields; the lower three panels show examples of nine cells with journey-dependent place fields that differed either by mean firing rate (second from top), spatial distribution (third panel from top), or both (bottom). The mean infield firing rate of journey-independent place fields (5.04 spikes/s) was higher then journey-dependent place fields (3.74 spikes/s; $t(370)=3.39, p<0.001)$, as would be expected if firing during preferred journeys was similar to journey-independent place fields, and decreased during nonpreferred journeys. $\boldsymbol{B}, \boldsymbol{C}$, The pie charts show proportions of journey-independent and journeydependent (prospective and retrospective) place fields in CA1 $(\boldsymbol{B})$ and CA3 $(\boldsymbol{C})$ during all STD sessions. The proportions were similar between CA1 and CA3.

0.85, $Z=3.5, p<0.001$, Fig. $4 C, E$; CA3: STD1, $r=0.5$, SW $1, r=$ $0.9, Z=4, p<0.001$, Fig. $4 D, F)$. Thus, the neuronal signal that distinguished corresponding journeys in the STD declined significantly in the SW as measured by both the proportion of journeydependent place fields and the spatial correlations of the sampled population.

Both memory accuracy and journey-dependent activity increased in the second SW sessions and were statistically indistinguishable from the STD (CA1: $\dot{\chi}_{(2)}=3.7, p=0.15$; CA3: $\dot{\chi}_{(2)}=$ $1.7, p=0.4$; Figure 4 ). CA1 firing rate correlations for the whole arms increased slightly, but not significantly, from the first to the second STD session ( $\mathrm{rSTD} 1 \mathrm{vs} \mathrm{rSTD} 2, z=1.8, p=0.07$ ). Journey coding was largely stable within the first SW session (proportion of journey-dependent place fields: first block of $5 \mathrm{SW}$ trials $=$ $26 \%$; second block of $5 \mathrm{SW}$ trials $=32 \%$; Fig. $5 \mathrm{~A}, B$ ).

\section{Journey coding was unstable across repeated SW sessions}

We hypothesized that journey codes formed via the same mechanisms that establish and stabilize place fields when rats first explore new environments, together with recurrent networks supported by CA3 or dentate mossy cell collaterals (Ferbinteanu and Shapiro, 2003). From this view, place and journey coding dynamics should be similar, i.e., whether or not place field analyses include journey comparisons. For example, standard measures of place field activity that ignore journeys should be stable as rats take familiar paths, and so should measures of journey dependence; both measures should change with similar dynamics during learning. Standard measures of place fields that ignored journey coding revealed stable CA1 and CA3 firing maps across repeated STD sessions, as quantified by categorical analysis, spatial correlations, firing rate indices, and population vector correlations (Bahar et al., 2011). Similarly, both journey-independent and journey-dependent fields were relatively stable in the same arm across repeated STD sessions in CA1 and CA3. Of these stable CA1 and CA3 place fields, 50\% maintained identical journey-dependent coding in both STD1 and STD2; equal proportions of the remaining fields switched between journey-independent and journeydependent coding from one session to the other in the same arm (Fig. 5D, stacked bars in left column). The population of active cells coding journeys was also stable in the STD. Eighty percent of the population of journey-dependent place fields in STD2 had journey-dependent place fields in STD1, and $18 \%$ of the journey-dependent population was active for the first time in STD2 (Fig. 5E, left column, stacked bars). Thus, measures of place coding and journey coding were relatively stable across repeated STD sessions and were equally common in CA1 and CA3 neurons.

As rats adapted to the SW task, however, place and journey coding dynamics dissociated. Although firing rate maps distinguished the two tasks, standard measures of place fields were as stable across repeated SW sessions as they were across repeated STD sessions (Fig. $8 A$; see Bahar et al., 2011, their Figs. 3-5). Thus, the mean spatial correlation of all CA1 place fields was similar across STD and SW sessions $(\sim 0.5$ and 0.4 , respectively, $p>0.36$ ). These standard measures compare only the spatial distributions of firing rates, however, and ignore other sources of variance, e.g., related to behavioral history. Comparing journeys revealed key dynamics of hippocampal coding. Journey coding was unstable across SW sessions. While $50 \%$ of cells maintained identical journey-dependent place fields in both STD sessions, only $17 \%$ of the cells active in the same arm in both SW sessions did so; most (83\%) switched between journeyindependent and journey-dependent firing patterns (Fig. 5D). In contrast to the $80 \%$ of the neural population with journeydependent place fields in both STD sessions, only $60 \%$ of the journey-dependent place fields in SW2 were recorded from cells that were active in SW1 (Fig. 5E). The proportion of cells with stable journey coding was significantly higher in repeated STD sessions than repeated SW sessions (stable fields: $\dot{\chi}_{(2)}=12, p=$ 0.002 ), and the proportion of new journey-dependent place fields was lower in STD2 than in SW2: $\dot{\chi}_{(1)}=7, p=0.008$; Figure $5 E$ ). 
Across repeated SW sessions, journeydependent activity remapped, both by switching between journey-independent, prospective, and retrospective coding categories and by partial global remapping in which newly active cells fired in journeydependent place fields. The results suggest that different mechanisms control where and in which behavioral sequences hippocampal neurons fire, which was verified by the opposite dissociation in the ALT task described next.

\section{Consistent proportions of journey-dependent place fields were maintained in the ALT task}

We had predicted that journey coding would be required for recent memory performance, that its prominence would correlate with overall memory performance, and that it should decline during error trials. Indeed, both journey coding and memory performance declined in the SW task. Although overall memory performance was far worse in the ALT than in the SW, the proportion of journeydependent place fields was maintained during correct trials. During the first ALT session, the rats responded to the unfamiliar environment by occasionally performing less reliably and exploring the new cues (sniffing, rearing, etc.). Because assessing journey-dependent activity requires identical overt behavior in the start and goal arms, many ALT trials were behaviorally unreliable and were excluded from further analysis. During the same sessions, however, other trials included reliable trajectories (i.e., equivalent location, heading, and speed statistics) that we analyzed. In these correct trials, the proportions of cells with journey-dependent activity were equivalent in the STD and the ALT in both neuronal populations $\left(\mathrm{CA} 1: \chi_{(2)}^{2}=2, p=0.3\right.$; CA3: $\chi_{(2)}^{2}=0.6, p=0.75$; Figure $6 A, B)$. Because fewer than 15 CA3 cells had place fields in the ALT sessions, these were excluded from subsequent analyses (Bahar et al., 2011; Fig. 6B). Spatial correlations between corresponding journeys were also equivalent during the first STD and ALT sessions when the entire population of CA1 place fields was considered (J1 vs J2, mean $r \pm$ SEM: STD1 $=0.5 \pm 0.04$, ALT1 $=$ $0.59 \pm 0.03, F_{(3,415)}=0.3, p=0.8$; mean rates collapsed across each arm, $r$ : STD1 $=0.69$, ALT1 $=0.73, Z=0.6, p=0.6$, Fig. $6 C, D)$. Together, the results show conclusively that the relative proportion of journey-dependent place fields does not predict overall memory performance.

To determine how rapidly journey-dependent activity developed with experience, we compared the first and last block of five correct trials in the first ALT session. From the first block, as soon as the rat made reliable trajectories and despite poor overall memory performance, journey-dependent coding emerged, with similar proportions of prospective and retrospective place fields as in the STD sessions $\left(\chi_{(1)}^{2}\right)=1.3, p=0.25$; Fig. 7). The journey coding did not require many trajectories in the new environment.
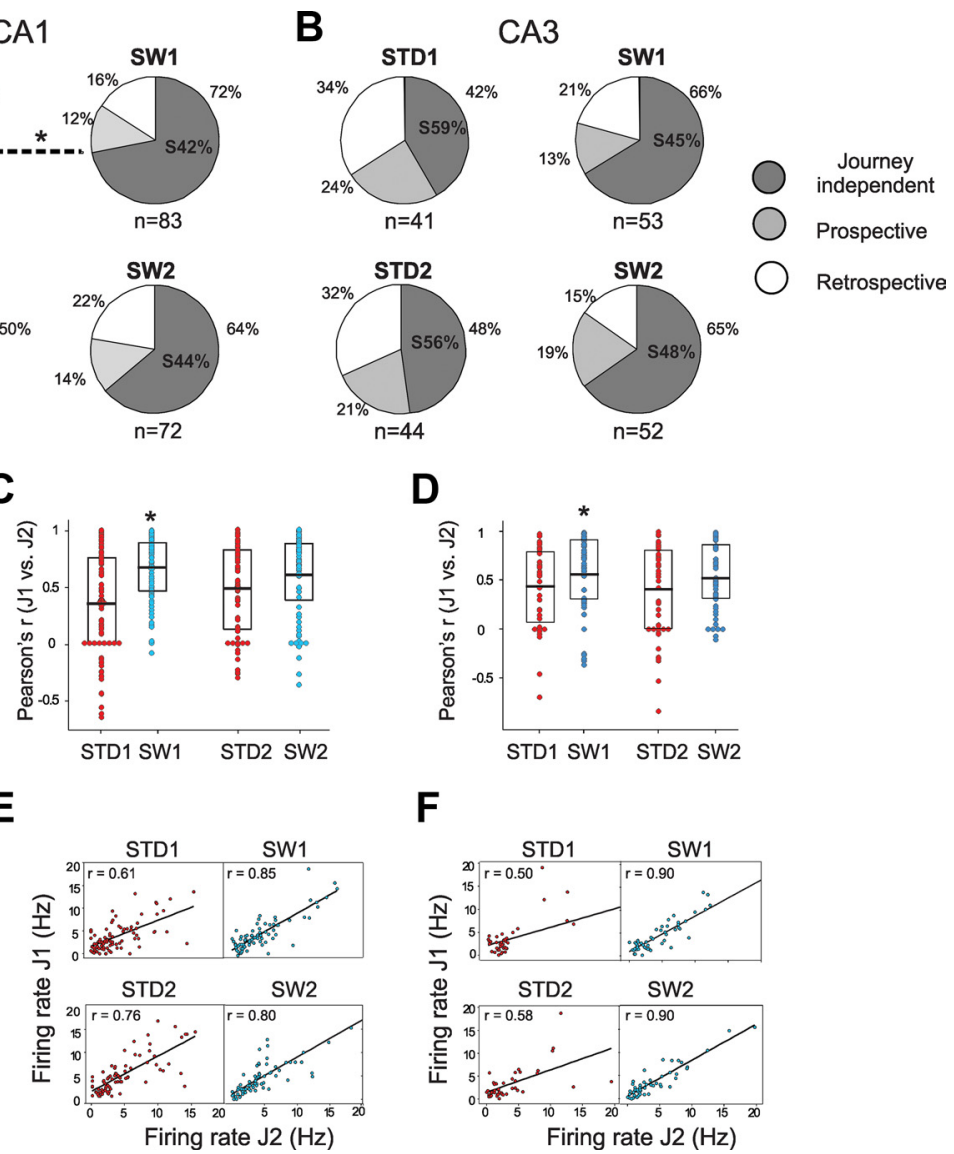

Figure 4. Journey-dependent activity during the SW task. $A, B$, The proportion of cells with journey-dependent activity declined during SW1 compared with STD1 in both CA1 $(\boldsymbol{A})$ and CA3 $(\boldsymbol{B})$, although the decline was statistically significant only in CA1 (me dotted line). Journey-dependent activity was separated into prospective and retrospective independent area (S\%). The number of total active cells included in each dataset is indicated below each pie. The decline in between journeys (J1 and J2) were significantly greater during SW1 than STD1 when the mean firing rates was calculated for each maze arms (collapsing grid units) in both $C A 1(\boldsymbol{E})$ and $C A 3(\boldsymbol{F})$.

On average, the rats made three errors and one meandering entry into the correct arm trial before the first block of behaviorally reliable correct trials was recorded.

\section{Journey coding was maintained even as most CA3 cells were silent and CA1 place fields were unstable}

We had proposed that journey-dependent activity was produced by the sequential activation of established place fields and predicted that the formation of stable place field maps should precede journey coding (Ferbinteanu et al., 2006). During the ALT sessions, however, the proportion of journey-dependent place fields was maintained well before the population of active CA1 cells had formed stable place field maps. Across repeated STD sessions, when memory performance was excellent $>70 \%$ of CA1 place fields occupied the same arm, and similar levels of stability accompanied repeated SW sessions. In contrast, only $47 \%$ of CA1 cells had place fields in the same arm in both ALT sessions, and $45 \%$ of the place fields were recorded only in ALT2 (Fig. 8A; Bahar et al., 2011, their Figs. 7-9). The unstable spatial representations were verified by converging categorical analyses, firing rate indices, and population vector correlations (Bahar et al., 2011). The place field dynamics varied with experience in the 
A
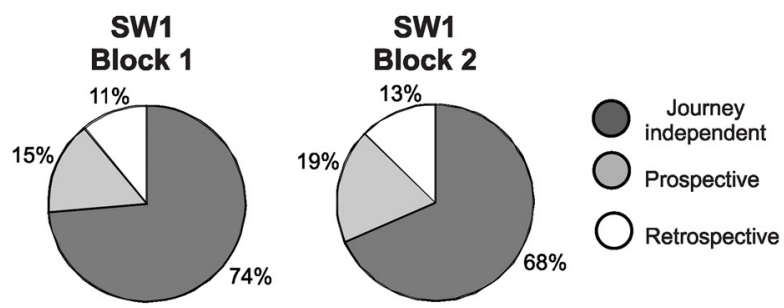

C
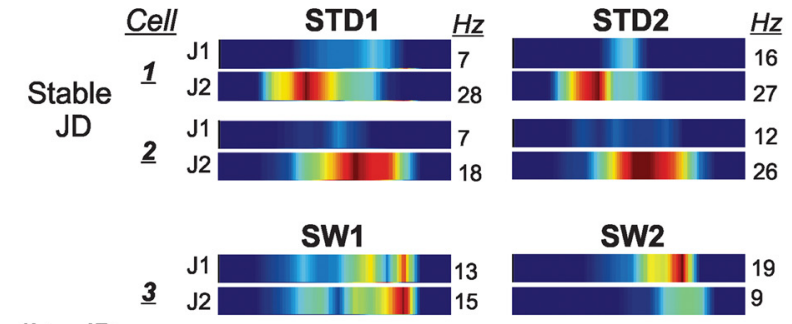

JI to JD

4
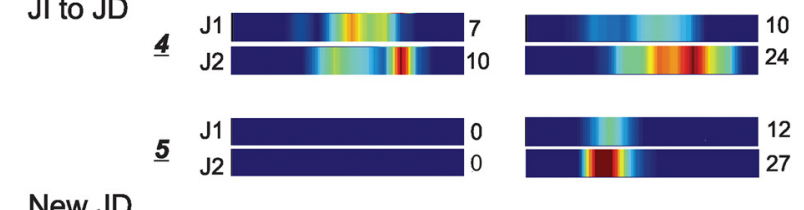

New JD

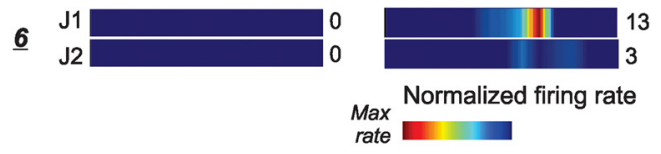

B

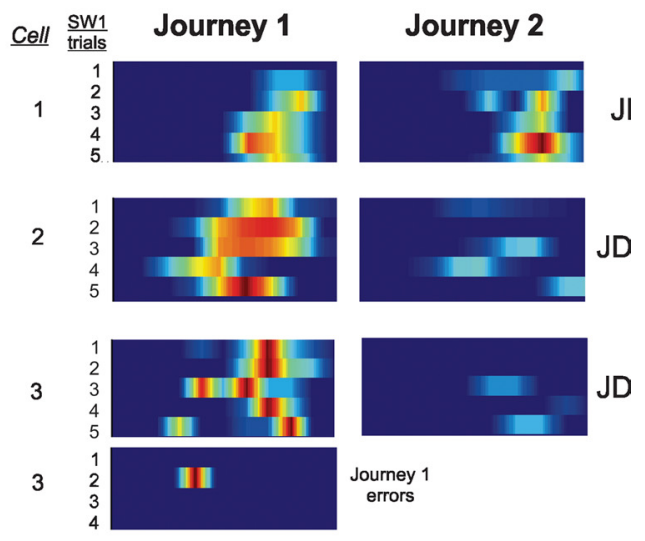

D

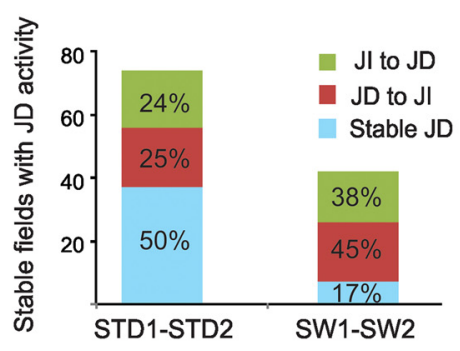

E

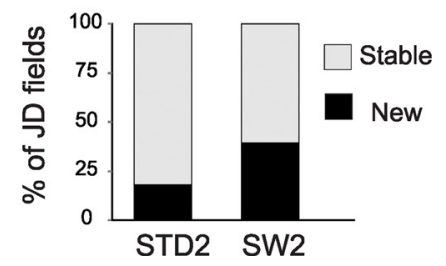

Figure 5. Journey dependent activity was relatively rare and unstable during SW1. A, Neural data were divided into the first and last block of $4-5$ correct trials in a SW recording session. Performance was stable between the first and second half of the session (correct and error trials included; percentage correct: block $1=81.7$, block $2=82.7$ ). Proportions of cells that had journey-dependent activity increased slightly between the first and second block of 5 correct SW trials. $B$, The firing rate maps show spiking activity of three CA1 cells during the first 10 correct and behaviorally reliable SW trials. The lowest panel shows activity of cell 3 during four error trials. JD, Journey dependent; Jl, Journey independent. C, Examples of JD activity dynamics across sessions. The firing rate maps show activity between journeys (J1 and J2) in repeated STD and SW sessions. The plurality of cells had stable JD activity between STD sessions (Stable JD). Many cells had different journey-related activity across repeated SW sessions (JI to JD, where JI is journey independent), while other cells inactive during SW1 had JD place fields in SW2 (New JD). D, The number of place fields that were identified in both repeated sessions (stable place fields) and were journey dependent in that at least one session was higher across repeated STD than SW sessions, indicated by the height of the stacked bars (STD1 versus STD2, left; SW1 versus SW2, right). The smaller height of the SW bars reflects the decline of journey coding in this task rather than a reduction in the number of place fields. The fate of these stable place fields with respect to journey coding is shown by the colors in the stacked bars (green: Jl to JD; red: JD to Jl; aqua: stable JD). In the STD, 50\% of the stable place fields that showed journey coding in either session maintained the same type of journey coding across both sessions; in the SW only $17 \%$ maintained the same journey coding across sessions. Stable JD place fields were more common in STD than SW sessions $\left(\chi_{(2)}^{2}=13, p=0.002\right)$. $E$, The stacked columns show the proportion of journey-dependent place fields recorded in the second session that had a place field of any type in the first session. The gray stack shows the proportion of cells with journey-dependent place fields in STD2 that also had place fields in STD1; the black stack shows the proportion of cells that had no place field in STD1 (left column). These proportions were significantly different across repeated SW sessions (right column). New JD place fields were significantly more common in the SW2 than the STD2 session $\left(\chi_{(1)}^{2}=7, p=0.008\right)$.

tasks and were not caused by electrode movement. Many of the same units that had stable place fields in repeated STD sessions had unstable place fields in the ALT (Bahar et al., 2011). Across repeated ALT sessions, CA1 cells with both stable and unstable place fields were recorded simultaneously from the same tetrodes, and both were distributed across probes (Fig. $8 \mathrm{~B}$ ). Moreover, place field stability was predicted by high mean infield firing rates during the first ALT session (mean spikes/s \pm SEM; ALT1: stable place fields $=8 \pm 0.6 \mathrm{~Hz}$, unstable place fields $=5.9 \pm 0.5$ $\mathrm{Hz}, p=0.011$ ), consistent with prior reports (Karlsson and Frank, 2008). The different firing rates occurred despite equivalent firing probability from one trial to another. Cells with stable and unstable place fields were equally likely to fire in a given trial (probability of spikes $>0$ per trial: stable place fields $=0.84$; unstable place fields $=0.74, t(20)=0.78, p=0.44)$.
We were surprised to find that the proportion of journeydependent place fields was maintained even as new CA1 firing maps emerged. The same proportion of journey-dependent place fields appeared in the first five correct ALT trials as in STD sessions, and the proportion was unchanged across ALT sessions as performance improved (Fig. 6). Moreover, the proportion of journey-dependent place fields was similar in both stable and unstable place fields (distribution of journey coding, stable vs unstable place fields: $\chi_{(2)}^{2}=0.6, p=0.7$; Fig. $8 C$ ). The strong and persistent journey signal was maintained even as the active population of CA1 cells changed: $45 \%$ of the place fields recorded in ALT2 were new, and 52\% of these new place fields were journeydependent (Fig. $8 F, G$ ). In other words, as rats performed a familiar task in an unfamiliar environment, CA1 activity reliably distinguished between corresponding journeys even as global re- 
mapping was ongoing, before stable place field maps were established. Furthermore, the same proportions of journeydependent place fields were maintained by CA1 neurons in the ALT and STD tasks despite a dramatic decline in CA3 place fields in the ALT (Bahar et al., 2011). The results imply that the formation of stable spatial maps and the selective activation of place fields within different behavioral sequences require different mechanisms, and that journey-selective activity does not depend upon CA3 collaterals.

\section{Journey coding declined during error trials}

The results described so far included only correct ALT trials, because most of the trials with reliable trajectories were also correct memory discriminations. Previous experiments reported that journey-dependent CA1 activity covaried with memory demand (Smith and Mizumori, 2006) and performance (Ferbinteanu and Shapiro, 2003), suggesting that it might reflect taskrelated memory processing. Although the relatively high number of errors in the first ALT session promised a strong test of this hypothesis, most trials included highly variable overt behavior and had to be excluded. We did not observe CA3 place fields during error trials in the ALT task, probably because most CA3 neurons were silent. Nonetheless, of the 18 journey-dependent CA1 place fields that met the strict behavioral criteria during both correct and error trials in the ALT (in three rats), 13 had altered responses during errors, a higher proportion than expected by chance $\left(\chi_{(1)}^{2}=23.8, p<\right.$ $0.01)$. Most $(n=10)$ place fields lost activity during what normally were the "preferred journeys," other place fields $(n=5)$ were unchanged during errors, and a few $(n=3)$ miscoded the journeys, e.g., by firing with the same high rate in the start arm during errors as during correct entries to the same goal arm. Journey coding was lost during errors in the ALT task even as overall firing rates were similar in both trial types (mean infield rates, spikes/s: correct = error trials $=\sim 3$; Bahar et al., 2011). Journey coding also declined during errors in the SW. Of the 11 journey-dependent CA1 place fields recorded during error trials in the SW, 6 lost journey coding $\left(\chi_{(1)}^{2}=6.5, p<0.02\right)$. In the SW, however, error trials were also accompanied by lower infield firing rates overall (mean infield rates, spikes/s: correct $=2.9$, error $=0.9$; Bahar et al., 2011). The dissociation of firing rate and journey-related activity changes during errors between the tasks provides further evidence that different mechanisms influence these firing properties. Intuitively, both place and journey signals should be required for successful performance in a spatial memory task, and both signals should decline simultaneously if error trials reflect a general retrieval failure. The results show that the two signals can dissociate and suggest that memory errors can occur if either place or journey coding fails. Future experiments that systematically vary error rate while maintaining overt behavior are these analyses.
B

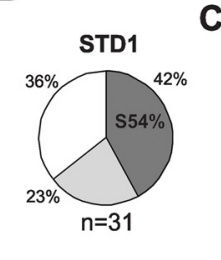

CA3
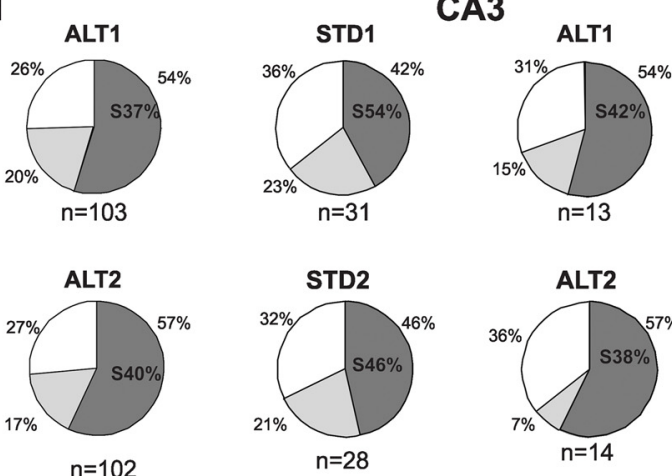

Journey
independent
Prospective

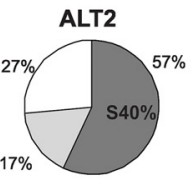

$\mathrm{n}=102$
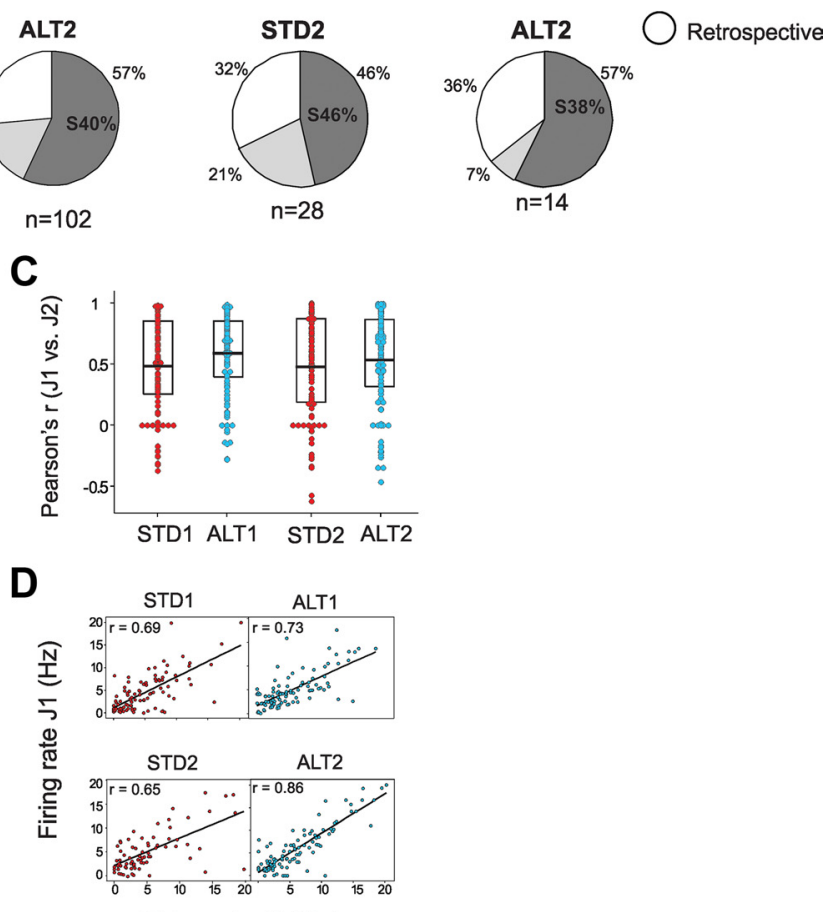

Firing rate $\mathrm{J} 2(\mathrm{~Hz})$

Figure 6. Journey-dependent activity was maintained during ALT trials. $\boldsymbol{A}, \boldsymbol{B}$, The pie charts show the proportions of CA1 ( $\boldsymbol{A})$ and $C A 3$ cells $(\boldsymbol{B})$ with journey-independent or journey-dependent activity (prospective or retrospective) in correct trials in STD and journey-independent place fields on the start arms is typed within the journey-independent area(S\%). The total number of active included in each dataset is indicated below each pie. The proportions of cells with journey-independent and journey(n) low number of CA 3 cells active during the correlation of firing rates on the arms between journeys was similar between the all STD and ALT sessions. D, The mean firing rates STD2 than ALT2 ( $r$ STD2 $=0.65$, ALT2 $=0.86, Z=3.6, p<0.001$ ). Too few CA3 cells were active during the ALT task to support

needed to further characterize how journey coding contributes to memory (Deadwyler et al., 1996).

\section{Journey coding "remapped" across tasks}

We proposed that journey selective activity by CA1 cells represented sequences that link the start and end of distinct, and perhaps unique, behavioral episodes (Ferbinteanu et al., 2006; Shapiro et al., 2006). Alternatively, prospective and retrospective activity could signal the beginning and end of behavioral episodes more generally, providing a common code for episodic boundaries across tasks analogous to start and stop sequences in gene transcription. For example, if cells with prospective activity signaled the initiation of goal-directed actions generically, then the same cells should maintain prospective coding in both the STD and SW. To distinguish these possibilities, we identified CA1 cells that had a place field in both the STD and SW, even if the place field was not stable in the same arm; we then categorized each of these place fields in each task as journey-independent, prospec- 

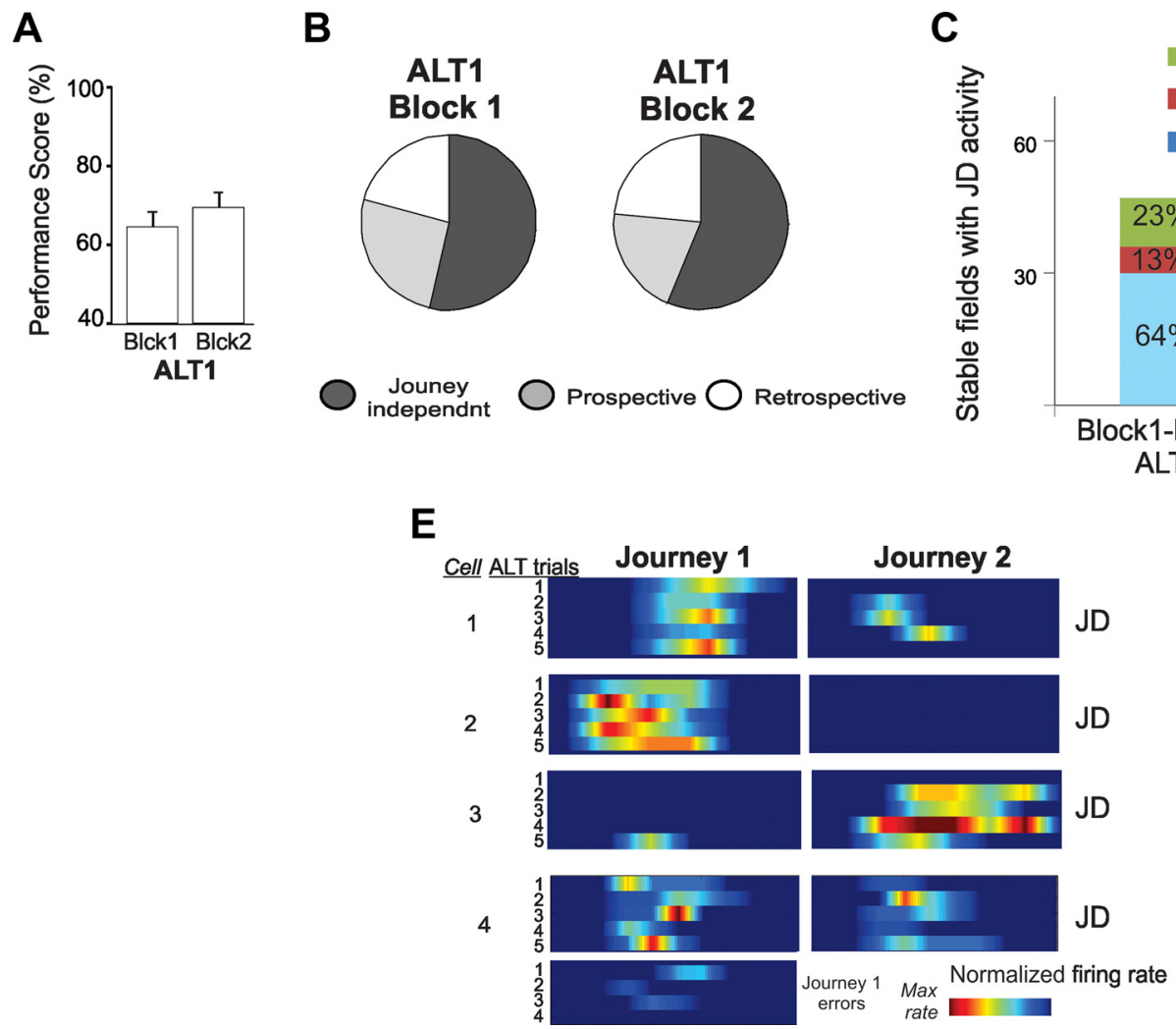

C

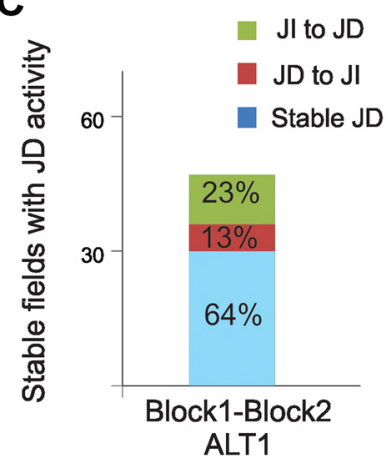

D

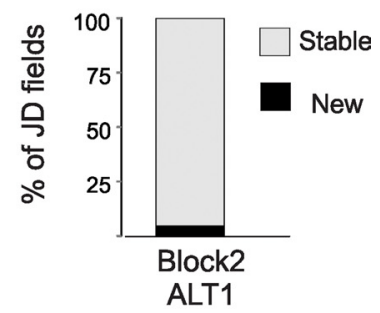

Figure 7. JD activity in CA1 was stable during the first and last block of 5 correct trials in ALT1. $A, B$, Memory performance $(\boldsymbol{A})$ and the proportions of journey-independent (JI) and journeydependent (JD) place fields $(\boldsymbol{B})$ were similar in both blocks. $\boldsymbol{C}$, The number of place fields that were identified in both blocks of the first ALT session (stable place fields) and were journey dependent in at least one block is indicated by the height of the stacked bar. Most place fields (64\%) maintained consistent journey coding (the blue portion of the stacked bar) despite the overall reduction in stable place fields compared to STD sessions. D, The stacked bar shows the proportion of journey-dependent place fields recorded in the second ALT block that had a place field of any type in the block. Few JD place fields (6\%) appeared for the first time in the second block of ALT1. E, Firing rate maps show the activity distribution of four CA1 cells during the first ten correct and behaviorally well structured ALT trials. The bottom panel shows the activity of cell 4 during error trials.

tive, or retrospective. We then counted the number of place fields that either maintained the same coding categories or switched among categories in two tasks. For example, if a cell had a journey-dependent place field in a start arm in both the STD and SW tasks, then it was categorized as maintaining prospective coding ( $p p$ in Fig. 9A). Note that preserved journey coding between the STD and SW would require place field activity in different maze arms in the two tasks.

Journey coding was not preserved, but rather tended to "remap" independently across tasks. About $30 \%$ of the CA1 units had journey-independent place fields in both the STD and SW tasks, and $<15 \%$ had the same journey-dependent coding category (Fig. 9A). About $45 \%$ of the CA1 units switched between journey-dependent and journey-independent place fields, and $<5 \%$ switched between prospective and retrospective. CA3 cells showed similar "journey remapping" patterns in the SW (Fig. $9 B$ ), as did both cell types between the STD and ALT tasks (Fig. $9 C, D)$. Thus, journey-independent place fields and journeydependent categories changed across tasks, showing that both coding properties are determined functionally rather than by fixed properties of different cell types.

\section{Discussion}

CA1 and CA3 neurons recorded in rats performing familiar and new variants of the same task revealed that place and journey coding followed different dynamics and predicted different aspects of learning and memory. Both CA1 and CA3 cells had stable journey-dependent and journey-independent place fields across
STD sessions, and both populations and coding categories "remapped" in the new tasks. In the SW, memory performance declined slightly, while place fields remapped immediately and stably. Journey-dependent activity initially declined significantly, was unstable between repeated SW sessions, and increased as performance improved. In the ALT, memory was markedly impaired initially, CA1 place fields were unstable, most CA3 place fields vanished, and performance improved as the place fields stabilized. Journey-dependent activity appeared immediately in the ALT in proportions equivalent to the STD before performance improved and were as common in new and unstable place fields as in stable place fields. Across tasks, consistent memory performance required stable place fields, and individual error trials were accompanied by reduced journey coding. Stable place field maps reflected familiarity with relationships among environmental features, while stable journey coding reflected familiarity with distinct, goal-directed behavioral sequences that generalized across environments. Together, place and journey coding signaled complementary information that linked new experiences with established memories, so that familiarity with either stimuli or behavioral strategies could inform learning.

\section{Journey-related activity and memory performance}

We proposed that journey-dependent activity in CA1 cells reflected a neural mechanism of episodic-like memory that represented temporally extended episodes and guided goal-directed memory discriminations (Ferbinteanu and Shapiro, 2003). From this view, journey codes linked the sequence of events from the 
A

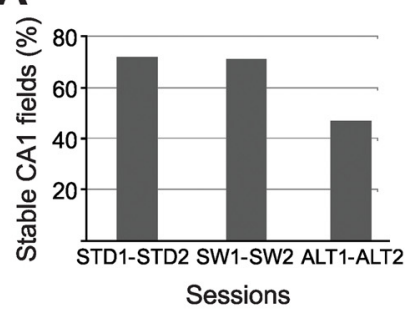

B

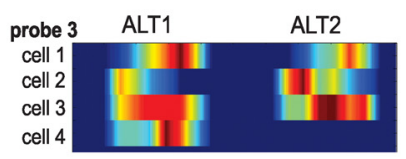

C

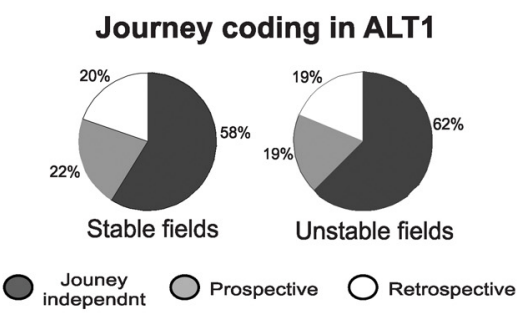

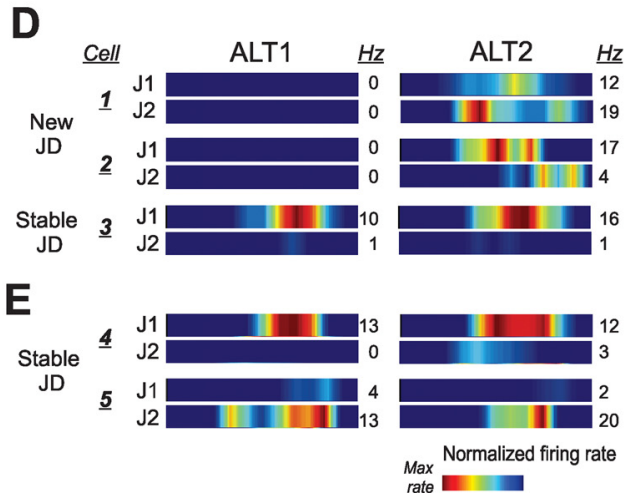

$\mathbf{F}$

G

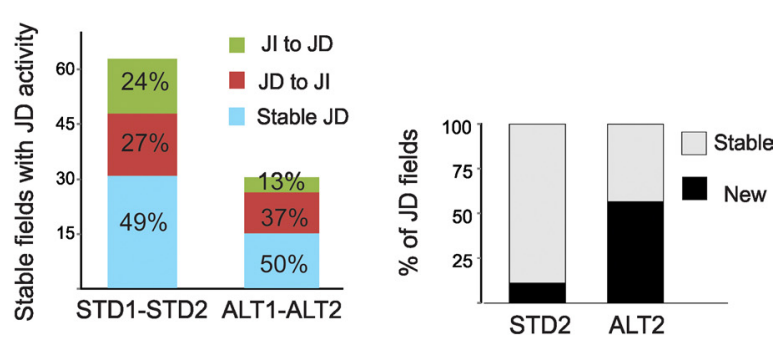

Figure 8. CA1 place fields remapped between ALT1 and ALT2 but journey coding remain strong. A, MostCA1 cells $(\sim 70 \%)$ had stable place fields on the same arm in repeated STD or SW sessions, but only $47 \%$ of the cells with place fields in ALT1 had place fields on the same arm in ALT2 (Bahar et al., 2011). B, Unstable and stable place fields were often recorded together by the same tetrode. C, The proportion of journey-dependent place fields recorded during ALT1 was similar in cells with stable and unstable place fields. $\boldsymbol{D}$, The firing rate maps show the activity of three cells recorded by the same tetrode across ALT1 and ALT2 sessions. Cells 1 and 2 fired on the waiting platform, were silent during ALT1, but had journey-dependent (JD) place fields in ALT2. Cell 3 had stable JD activity in both sessions. $\boldsymbol{E}$, Cells 4 and 5, recorded simultaneously from another tetrode, showed stable JD activity across ALT1 and ALT2. $F$, The number of place fields that were identified in both repeated sessions (stable place fields) and were journey-dependent in at least one session was higher across repeated STD (62) than ALT (30) sessions, indicated by the height of the stacked bars (STD1 versus STD2 (left) and ALT1 versus ALT 2 (right). The smaller height of the ALT bars reflects the decline of place field stability in this task rather than a reduction in the number of place fields per se. The fate of these stable place fields with respect to journey coding is shown by the colors (as described in caption 5D). Similar proportions of stable JD place fields were recorded across repeated STD and ALT sessions ( 50\%) even though the absolute number of stable place fields was greater in the STD. Jl, Journey independent. G, The stacked columns show the proportion of journeydependent place fields recorded in the second session that had a place field of any type in the first session. The gray stack in the left column shows the proportion of cells with journey-dependent place fields in STD2 that also had place fields in STD1; the black stack shows the proportion of cells that had no place field in STD1. These proportions were significantly different across repeated ALT sessions (right column), when many new place fields emerged (Bahar et al., 2011). Moreover, new JD codes were more common in newly emerged place fields in ALT 2 (25/48,52\%) than in the place fields that were stable across ALT1 and ALT2 $(4 / 19,21 \%)\left(\chi_{(1)}^{2}=5.3, p<0.03\right)$.

appetitive start to the consummatory goal of behavioral episodes and thereby provided a neuronal mechanism for episodic-like memory. We predicted that recent memory should require prominent journey coding that would correlate with overall performance, and that its decline should accompany errors (Ferbinteanu et al., 2006). The new results verified that journey coding is common in CA1 and discovered that it is equally common in CA3. Because journey coding occurred in all conditions, the results do not determine its necessity for memory performance. The new results show clearly that the proportion of journey coding cells does not predict overall memory performance, which was better in the SW than the ALT, while journey coding was more common in the ALT than the SW. Future experiments could determine whether memory is impaired when journey coding is disrupted completely. Individual error trials were typically associated with reduced journey coding (compare Ferbinteanu and Shapiro, 2003; Ferbinteanu et al., 2011), suggesting that the presence of journey coding, if not its proportion, contributes to memory accuracy.

\section{Journeys and error trials}

If memory performance requires activating hippocampal firing patterns that correspond to specific collections of task features (Komorowski et al., 2009), then errors should occur whenever firing patterns fail to do so. To perform the plus maze tasks, the rat had to remember the current goal and select one of two po- tential journeys in each trial, i.e., to represent "where" and "which." Journey coding declined during error trials in both SW and ALT tasks, while other coding failures distinguished errors in two tasks. The SW required new responses to familiar items (i.e., pattern separation), and the hippocampus generated distinct representations of the same environment. CA1 population vectors strongly distinguished the STD and the SW during correct trials, and memory errors occurred when this "anti-coding" diminished or firing rate declined (Bahar et al., 2011). The ALT task required applying familiar rules to unfamiliar places, and memory performance was poor until new, stable place fields were established. Memory errors in the ALT task occurred when journey coding failed, even as overall firing rates were unchanged (Bahar et al., 2011). Thus, matching-to-place required both place and journey coding, and errors occurred when either signal diminished.

\section{Journey coding is determined functionally}

We proposed that journey-dependent activity was a functional property of hippocampal neurons established during learning via activity-dependent synaptic plasticity (Kentros et al., 1998). Indeed, journey codes "remapped" between tasks and across SW sessions (Fig. 5D,E), suggesting that hippocampal activity represents specific sequences-the start and end of particular behavioral episodes. Journey dependence was clearly determined functionally, as many neurons with journey-independent place 
A
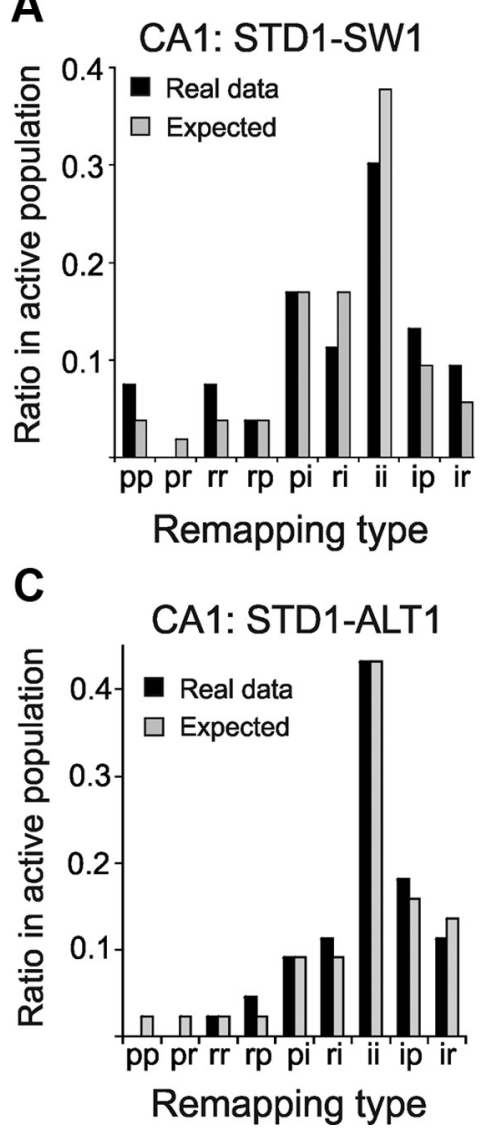

B

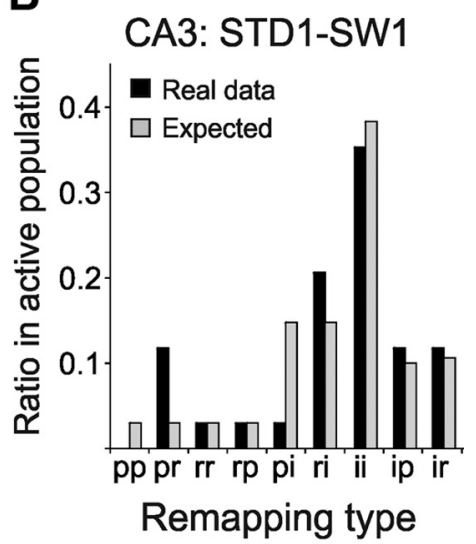

D

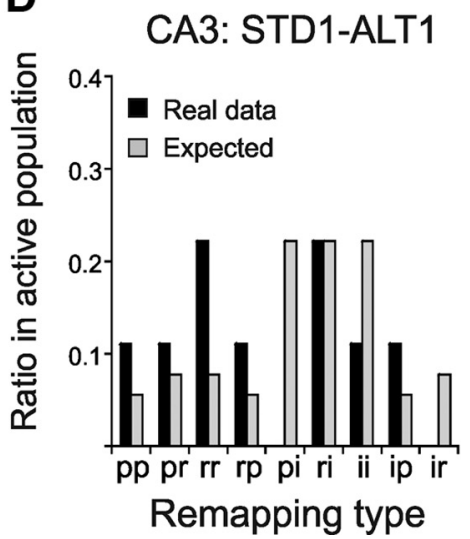

Figure 9. Journey coding categories remapped across tasks. The histograms show the distributions of journey-independent, prospective, and retrospective place fields in the new tasks (SW or ALT) in all cells with a place field in both the STD and the new task (independent of maze arm). The actual distributions based on the STD session (Real data, black bars) were compared to the distributions expected by independent coding across tasks (Expected, gray bars). The category labels on the $x$-axes describe, from left to right, journey coding in the STD and new tasks, respectively. For example, " $p p$ " includes cells that had prospective activity in both STD1 and the new task; "ir" includes cells that had journey-independent activity during the STD task and retrospective activity during the new task, etc.; $p$, prospective, $r$, retrospective; and $i$, journey independent. The actual and expected distributions were statistically indistinguishable. $\boldsymbol{A}$, STD and SW sessions, CA1: $\chi_{(7)}^{2}=3.2, p=0.86 ; \boldsymbol{B}, \mathrm{CA}_{\mathrm{O}}: \chi_{(7)}^{2}=4.8, p=0.67 ; \boldsymbol{C}$, STD and ALT sessions, $C A 1: \chi_{(5)}^{2}=0.6, p=0.99 ; D, C A 3$ : sign test $=3, p=0.73$ (too few samples for $\chi^{2}$ ).

fields in one task had journey-dependent place fields in another (Fig. 9).

Independent mechanisms influence place and journey coding We proposed that journey coding reflected the sequential activation of place fields via recurrent hippocampal circuits (McNaughton and Morris, 1987) and predicted that journey-independent place fields should form and stabilize either before or concurrently with journey-dependent place fields (Ferbinteanu and Shapiro, 2003; Ferbinteanu et al., 2006). The results show a double dissociation in the emergence and stability of place and journey coding, and their different dynamics suggest that they reflect different neuronal mechanisms. Standard measures of place fields calculated mean firing rates in locations independent of journeys and revealed different dynamics across repeated STD, SW, and ALT sessions. Place fields were stable in the STD, remapped immediately and stably in the SW, and gradually developed new maps in the ALT (Bahar et al., 2011). The new results showed that journey-related activity changed in both stable and unstable place fields. Place field maps were stable across repeated SW sessions, but the proportion of journey-dependent place fields declined and journey coding "remapped" with experience (Smith et al.,
2011). Place field maps were unstable across repeated ALT sessions (Bahar et al., 2011), but the proportion of journeydependent place fields was maintained and journey "remapping" continued. Journey-dependent activity did not emerge gradually or entail repeated trajectories but was present during the first five correct trials in the SW and the ALT tasks (Figs. 5, 7). The results also suggest that journey coding does not require CA3 activity. Similar numbers of CA1 cells had place fields in STD, SW, and ALT sessions, whereas CA3 cells were mostly silent in the ALT, when journey coding was prominent. The dissociations indicate that different circuits affect place field maps and journey codes.

\section{Strategies, reward expectancies, and journey coding}

The present findings suggest a neuropsychological model of memory-guided learning. We propose that during STD training, the rat forms a representation of the environment that integrates locations, task strategy, and reward expectancies. From this view, the hippocampal representation combines "where," "how," and "which." During STD training, hippocampal place fields encoded relationships among stimuli and movements within the environment ("where") via input from other medial temporal lobe structures (Brun et al., 2002; van Cauter et al., 2008). Other concurrent inputs signaled by medial and orbital prefrontal cortices (PFCs conveyed information about strategy ("how") and reward expectancy ("which"). Through synaptic plasticity, these inputs became associated with goal-directed actions: specific journeys through the environment. Subsequently, the different subsets of inputs could serve as content-addressable retrieval signals for the established relational representations (Eichenbaum et al., 1999). From this view, strategy and goal information provide "schemas" that activate hippocampal codes in the same way that incomplete subsets of environmental cues can activate normal place fields.

Recent evidence is consistent with this view. Memory strategy and reward expectancy signals are recorded in medial PFC (mPFC) and orbital PFC (OFC) and influence the hippocampus either directly or indirectly (Rich and Shapiro, 2009; Young and Shapiro, 2011). Switching between memory strategies requires mPFC and alters CA1 coding (Ferbinteanu et al., 2011). We propose that $\mathrm{mPFC}$ signals also inform CA1 codes about other abstract task features, such as matching-to-sample rules, cognitive structures that allow one place, e.g., a start arm, to be parsed and represented as a common path to different goals. In the plus maze, specific journeys are selected by the current goal, which changes across trial blocks. We propose that the goal is selected by reward expectancy signals originating in the OFC. Reversal learning requires the OFC, where neurons show "path coding" related to reward expectancies (Young and Shapiro, 2011). Together, 
mPFC neurons convey memory strategy and the matching-tosample rules to CA1 that parse different journeys ("how"), while OFC neurons convey reward expectancy signals that specify which particular journey leads to the current goal ("which"). Frontotemporal interactions thereby integrate relevant goals and strategies to particular situations.

This view helps interpret the dynamics of place and journey codes as well as the different learning rates in the SW and ALT tasks. In the STD task, strategies and reward expectancies converge on stable spatial representations as rats made familiar journeys to established goals. Training in the STD task facilitated learning (compared to naive animals) in both the SW and the ALT because both strategies and rules (e.g., matching-to-place) were identical. Performance in the new tasks declined initially compared to the STD because reward expectancies were discordant in the SW and environmental representations were insufficient in the ALT. From this view, the slight decline in SW performance and the steep decline in journey coding reflected the violation of reward expectancies and reduced coherence between the hippocampus and the OFC (Young and Shapiro, 2011). The relatively good performance in the SW reflected the rapid integration of the familiar strategy and environment. Hippocampal place fields reorganized in the SW compared to the STD, and the new maps were stable and maintained key discriminative stimuli: $56 \%$ of CA1 and $71 \%$ of CA3 neurons had place fields in both tasks, and $32 \%$ of CA1 and $62 \%$ of CA3 cells had place fields on the same arm in the STD and the SW (Bahar et al., 2011). Even as the population of active neurons with place fields was stable, journey-dependent activity changed, as though new reward expectancies computed by the OFC were integrated with a well established representation of strategy and place.

The more severe impairment in the ALT task and the marked instability of place fields reflected the formation of new hippocampal representations in an unfamiliar environment: $60 \%$ of CA 1 and $80 \%$ of the CA3 cells had place fields exclusively in either the STD or the ALT (Bahar et al., 2011). High proportions of journey-dependent place fields were maintained in the ALT despite the instability of the active CA1 population and the dramatic loss of CA3 place fields. We propose that journey-dependent activity was maintained in the ALT by familiar strategies that, unlike in the SW, were unencumbered by reversed contingencies. In other words, journey coding by CA1 may have been maintained by coherent interactions between the hippocampus and both the mPFC and OFC (Young and Shapiro, 2011). This interpretation is consistent with the striking loss of CA3 place fields in the ALT task, and the differential and powerfully excitatory projection from the prefrontal cortex to CA1, but not CA3, directly (Cenquizca and Swanson, 2007) and via the nucleus reunions (Vertes, 2006). Future experiments that record from both cell regions while different prefrontal cortical circuits are manipulated could test these possibilities.

\section{References}

Ainge JA, Tamosiunaite M, Woergoetter F, Dudchenko PA (2007) Hippocampal CAl place cells encode intended destination on a maze with multiple choice points. J Neurosci 27:9769-9779.

Bahar AS, Shirvalkar PR, Shapiro ML (2011) Memory guided learning: CA1 and CA3 neuronal ensembles differentially encode the commonalities and differences between situations. J Neurosci 31:12270-12281.

Bower MR, Euston DR, McNaughton BL (2005) Sequential-contextdependent hippocampal activity is not necessary to learn sequences with repeated elements. J Neurosci 25:1313-1323.

Brun VH, Otnass MK, Molden S, Steffenach HA, Witter MP, Moser MB, Moser EI (2002) Place cells and place recognition maintained by direct entorhinal-hippocampal circuitry. Science 296:2243-2246.
Cenquizca LA, Swanson LW (2007) Spatial organization of direct hippocampal field CA1 axonal projections to the rest of the cerebral cortex. Brain Res Rev 56:1-26.

Deadwyler SA, Bunn T, Hampson RE (1996) Hippocampal ensemble activity during spatial delayed-nonmatch-to-sample performance in rats. J Neurosci 16:354-372.

Eichenbaum H, Dudchenko P, Wood E, Shapiro M, Tanila H (1999) The hippocampus, memory, and place cells: is it spatial memory or a memory space? Neuron 23:209-226.

Ekstrom AD, Kahana MJ, Caplan JB, Fields TA, Isham EA, Newman EL, Fried I (2003) Cellular networks underlying human spatial navigation. Nature 425:184-188.

Ferbinteanu J, Shapiro ML (2003) Prospective and retrospective memory coding in the hippocampus. Neuron 40:1227-1239.

Ferbinteanu J, Kennedy PJ, Shapiro ML (2006) Episodic memory - from brain to mind. Hippocampus 16:691-703.

Ferbinteanu J, Shirvalkar P, Shapiro ML (2011) Memory modulates journeydependent coding in the rat hippocampus. J Neurosci 31:9135-9146.

Frank LM, Brown EN, Wilson M (2000) Trajectory encoding in the hippocampus and entorhinal cortex. Neuron 27:169-178.

Gelbard-Sagiv H, Mukamel R, Harel M, Malach R, Fried I (2008) Internally generated reactivation of single neurons in human hippocampus during free recall. Science 322:96-101.

Hampson RE, Deadwyler SA (2000) Cannabinoids reveal the necessity of hippocampal neural encoding for short-term memory in rats. J Neurosci 20:8932-8942.

Ji D, Wilson MA (2008) Firing rate dynamics in the hippocampus induced by trajectory learning. J Neurosci 28:4679-4689.

Karlsson MP, Frank LM (2008) Network dynamics underlying the formation of sparse, informative representations in the hippocampus. J Neurosci 28:14271-14281.

Kennedy PJ, Shapiro ML (2009) Motivational states activate distinct hippocampal representations to guide goal-directed behaviors. Proc Natl Acad Sci U S A 106:10805-10810.

Kentros C, Hargreaves E, Hawkins RD, Kandel ER, Shapiro M, Muller RV (1998) Abolition of long-term stability of new hippocampal place cell maps by NMDA receptor blockade. Science 280:2121-2126.

Komorowski RW, Manns JR, Eichenbaum H (2009) Robust conjunctive item-place coding by hippocampal neurons parallels learning what happens where. J Neurosci 29:9918-9929.

Lenck-Santini PP, Fenton AA, Muller RU (2008) Discharge properties of hippocampal neurons during performance of a jump avoidance task. J Neurosci 28:6773-6786.

Leutgeb S, Leutgeb JK, Treves A, Moser MB, Moser EI (2004) Distinct ensemble codes in hippocampal areas CA3 and CA1. Science 305:1295-1298.

Markus EJ, Qin YL, Leonard B, Skaggs WE, McNaughton BL, Barnes CA (1995) Interactions between location and task affect the spatial and directional firing of hippocampal neurons. J Neurosci 15:7079-7094.

McNaughton BL, Morris RGM (1987) Hippocampal synaptic enhancement and information storage within a distributed memory system. Trends Neurosci 10:408-415.

Moita MA, Rosis S, Zhou Y, LeDoux JE, Blair HT (2003) Hippocampal place cells acquire location-specific responses to the conditioned stimulus during auditory fear conditioning. Neuron 37:485-497.

Muller RU, Kubie JL (1987) The effects of changes in the environment on the spatial firing of hippocampal complex-spike cells. J Neurosci 7:1951-1968.

O'Keefe J, Nadel L (1978) The hippocampus as a cognitive map. Oxford: Oxford UP.

Paz R, Gelbard-Sagiv H, Mukamel R, Harel M, Malach R, Fried I (2010) A neural substrate in the human hippocampus for linking successive events. Proc Natl Acad Sci U S A 107:6046-6051.

Rich EL, Shapiro M (2009) Rat prefrontal cortical neurons selectively code strategy switches. J Neurosci 29:7208-7219.

Robbe D, Buzsáki G (2009) Alteration of theta timescale dynamics of hippocampal place cells by a cannabinoid is associated with memory impairment. J Neurosci 29:12597-12605.

Schacter DL, Addis DR (2009) On the nature of medial temporal lobe contributions to the constructive simulation of future events. Philos Trans R Soc Lond B Biol Sci 364:1245-1253.

Shapiro ML, O'Connor C (1992) N-methyl-D-aspartate receptor antagonist 
MK-801 and spatial memory representation: working memory is impaired in an unfamiliar environment but not in a familiar environment. Behav Neurosci 106:604-612.

Shapiro ML, Kennedy PJ, Ferbinteanu J (2006) Representing episodes in the mammalian brain. Curr Opin Neurobiol 16:701-709.

Smith DM, Mizumori SJ (2006) Learning-related development of contextspecific neuronal responses to places and events: The hippocampal role in context processing. J Neurosci 26:3154-3163.

Smith DM, Barredo J, Mizumori SJY (2011) Complementary roles of hippocampus and retrosplenial cortex and behavioral context discrimination. Hippocampus. Advance online publication. Retrieved May 21, 2011. doi:10.1002/hipo.20958.

Squire LR, Stark CE, Clark RE (2004) The medial temporal lobe. Annu Rev Neurosci 27:279-306.
SYSTAT Software (2007) SYSTAT 12: Statistics I-IV manual. San Jose, CA: SYSTAT Software.

Tulving E, Markowitsch HJ (1998) Episodic and declarative memory: role of the hippocampus. Hippocampus 8:198-204.

Van Cauter T, Poucet B, Save E (2008) Unstable CA1 place cell representation in rats with entorhinal cortex lesions. Eur J Neurosci 27:1933-1946.

Vertes RP (2006) Interactions among the medial prefrontal cortex, hippocampus and midline thalamus in emotional and cognitive processing in the rat. Neuroscience 142:1-20.

Wood ER, Dudchenko PA, Robitsek RJ, Eichenbaum H (2000) Hippocampal neurons encode information about different types of memory episodes occurring in the same location. Neuron 27:623-633.

Young JJ, Shapiro ML (2011) Dynamic coding of goal-directed paths by orbital prefrontal cortex. J Neurosci 31:5989-6000. 